\title{
Alcohol and Drug Use During Sexual Activity and Compliance with Safe Sex Guidelines for AIDS: The AIDS Behavioral Research Project
}

\author{
Ron Stall, PhD, MPH \\ Leon McKusick, PhD \\ James Wiley, PhD \\ Thomas J. Coates, PhD \\ David G. Ostrow, MD, PhD
}

This article describes the association between drug and alcohol use during sexual activity and high-risk sex for AIDS. Data to test this association are drawn from a prospective study of the behavioral changes made by gay men in San Francisco in response to the AIDS epidemic. Findings drawn from the May, 1984 and May 1985 waves of data collection are described. The cross-sectional analysis showed that use of particular drugs during sex, the number of drugs used during such activity, and the frequency of combining drugs and sex are all positively associated with risky sexual activity for AIDS. The retrospective data showed that men who currently abstained from combining drug use with sexual activity were likely to have been at no risk for AIDS over two measurement points during the previous year. The men who currently combined drug use with sex were most likely to have a history of high-risk sexual activity over the previous year. These findings show a strong relationship between drug and alcohol use during sex and non-compliance with safe sex techniques to prevent the spread of AIDS. Implications of this relationship for AIDS health education efforts are discussed.

\section{INTRODUCTION}

The fact that, at present, there is neither a cure nor a vaccine for AIDS has lent tremendous importance to health education efforts designed to prevent the spread of this disease. The efficacy of health education efforts is demonstrated by the high

Ron Stall is with the Alcohol Research Group, Medical Research Institute of San Francisco, 1816 Scenic Avenue, Berkeley CA 94709 and Department of Urban Studies, Rutgers University, New Brunswick, NJ 08903.

Leon McKusick and Thomas J. Coates are with Division of General Internal Medicine, University of California, San Francisco CA 94143.

James Wiley is with the Survey Research Center, University of California, Berkeley CA 94720.

David G. Ostrow is with the Department of Psychiatry, School of Medicine and Institute for Social Research, University of Michigan, Ann Arbor MI 48109. 
degree of correct identification among gay male populations of the sexual behavior thought likely to spread the HTLV-III virus, ${ }^{1}$ as well as lowered rates of participation in such sexual practices over time. ${ }^{2}$ However, the study of the behavioral changes made by gay men in response to the AIDS epidemic has also demonstrated that compliance with health education guidelines has been uneven. That is, some men have been able to minimize their risk for sexual exposure to the HTLV-III virus through careful compliance with health education recommendations. while other men have been less successful in consistently restricting their sexual expression to the bounds introduced by the recommendations of health educators. This finding raises the question of whether certain circumstances (or sets of circumstances) can be identified under which compliance with health education recommendations is suspended.

This article will determine whether an association exists between alcohol and/or drug use during sexual activity and the adoption of "safe sex" techniques (e.g., sexual behaviors thought not likely to transmit the HTLV-III virus). This question is warranted not only by the very high prevalence rates for problematic alcohol and drug use reported for convenience samples of gay males. ${ }^{3-5}$ but also by the literature which has investigated the effects of alcohol consumption (or beliefs about alcohol consumption) on sexual arousal. ${ }^{6-11}$ Implications of the associations between high-risk sexual activity and drug and alcohol use for furthering the efficacy of health education efforts will be discussed.

\section{METHODOLOGY}

Data reported in this article are drawn from a three-year prospective study of the changes in sexual behavior made by gay men in San Francisco in response to the AIDS epidemic. ${ }^{1.2}$ This research project was originally designed to determine how the AIDS epidemic was influencing gay men's psychological adjustment and sexual behavior. A survey questionnaire was administered semiannually since November, 1983 to four groups of sexually active gay men in San Francisco. These men were recruited in the following ways:

Bathouse Group - 400 men were invited to participate in this study by giving them questionnaires as they left three popular bathhouses between 8 p.m. and 2 a.m. 151 men from this group returned completed questionnaires in a form suitable for data entry.

Bar Group - 400 men were invited to participate in this study by giving them questionnaires as they left gay bars in several distinct gay neighborhoods between 6 p.m. and 2 a.m. 134 men from this group returned completed questionnaires in a form suitable for data entry.

No Bar/No Bath Group-250 men who frequented neither gay bars nor bathhouses for the purposes of making sexual contacts during the previous 2 month period were recruited through advertising in local gay newspapers and fliers sent to gay professionals and organizations. 181 men from this group returned completed questionnaires in a form suitable for data entry.

Couples Group-500 men (250 gay couples) who had participated in other investigations received a letter and questionnaire requesting participation in this study. 189 men from this group returned completed questionnaires in a form suitable for data entry. 
Taking opportunistic samples from relatively distinct subgroups of gay men was undertaken to maximize the potential for constructing a balanced picture of the types of changes in sexual behavior that gay men in general have made in response to the AIDS epidemic. This method of recruitment resulted in an initial group of participants with higher overall numbers of sexual partners than were found in two separate probability samples of gay men in San Francisco, ${ }^{12,13}$ particularly for men not involved in relationships. However, the behavior reported by the ongoing cohort by May, 1985 closely approximated that reported by a random telephone survey of gay and bisexual men in San Francisco at about the same time. ${ }^{14}$ Thus, data elicited from this sample are a useful measure of behavioral adaptations that have occurred within the gay male community of San Francisco.

During the May, 1985 wave of data collection a set of items was included in the questionnaire which measured whether respondents had "never, rarely, sometimes, or often" drank alcoholic beverages, used amyl or butyl nitrates "poppers", smoked marijuana or hashish, or used "other drugs" while having sex during the previous month. These items were only asked of the men not in monogamous relationships or who had sex outside of this primary relationship $(n=463)$ and were not administered during the May, 1984 wave of data collection. A primary relationship was one involving commitment to another individual over anyone else and one that had a sexual component. Men in monogamous gay relationships were excluded from the analysis since participation in sexual activity within the bounds of such relationships was unlikely to result in further HTLV-III infection.

As was true during all of the waves of data collection, a detailed measure of participation during the past 30 days in a wide variety of sexual behaviors was also administered. The risk score was based on information disseminated by health education campaigns conducted in San Francisco during the study period. That is, sexual behavior which was considered completely safe was assigned a weight of 0 (e.g., massage, cuddling, frottage). Sexual behavior which was considered "probably safe" was assigned a weight of 1 (e.g., wet kissing, anal intercourse with condoms, oral sex, water sports, and digital penetration), those "probably risky" a weight of 2 (e.g., rimming, swallowing semen during oral sex, and penetration with a dildo), and behavior thought to be "risky" was assigned a weight of 3 (e.g., anal intercourse without a condom and fisting). The risk score was derived by multiplying frequency of each risk behavior during the previous month by the assigned weight, and then summing these products. Risk scores were divided for the analysis into "no risk," "medium risk," and "high risk." Those at "no risk" had scores of $0(n=117)$, those at "medium risk" had scores of $1-9(n=171)$, and those at high risk had scores of 10 or higher $(n=175)$. Men with risk scores higher than 1 were divided roughly in half, so that the distributions for the risk scores were roughly trichotomized. The same sexual risk items were asked of the same men during the May, 1984 and May, 1985 waves of data collection, allowing an accurate estimate of changes in sexual behavior over time. The same summary risk score was used for the May, 1984 data as for the May, 1985 data.

\section{RESULTS}

In Table 1 the relationship between drinking alcohol during sexual activity and participation in high risk sexual activity is summarized. Men who never drank during sex 
Table 1. Frequency of Drinking While Having Sex by Risk Scores for AIDS, In Percentages

While Having Sex Last Month How Often Had You Been Drinking?

\begin{tabular}{lcccc} 
& $\begin{array}{c}\text { Never } \\
(n=165)\end{array}$ & $\begin{array}{c}\text { Rarely } \\
(n=73)\end{array}$ & $\begin{array}{c}\text { Sometimes } \\
(n=122)\end{array}$ & $\begin{array}{c}\text { Often } \\
(n=103)\end{array}$ \\
\hline Risk Score for AIDS & & & & \\
No Risk & 43.6 & 15.1 & 16.4 & 13.6 \\
Medium Risk & 34.5 & 38.4 & 37.7 & 38.8 \\
High Risk & 21.8 & 46.6 & 45.9 & 47.6 \\
Total & $100 \%$ & $100 \%$ & $100 \%$ & $100 \%$ \\
Chi square $=52.2, p=.0000$ & & & \\
\hline
\end{tabular}

were about 3 times more likely to be in the no risk group than those who combined drinking with sex at any other frequency level. Conversely, the men who drank even rarely during sexual activity were about 2 times as likely to be at high risk as those who abstained from alcohol during sex. It seems that any combination of drinking and sexual activity is associated with high risk behavior. This relationship is highly significant.

The relationship between the prevalence of use of a particular drug during sexual activity at least once during the past 30 days (i.e., combining "rarely," "sometimes," or "often" used during sex) and participation in risky sexual behavior for AIDS is described in Table 2. Subtracting the figures in each cell from $100 \%$ gives the proportion of men at each risk level who did not use a particular drug during sexual activity over the past 30 days. Depending on the specific drug, the men at high risk are from 2 to 3.5 times more likely to have used drugs during sexual activity than the men at no risk. The men at medium risk are approximately 1.5 times more likely to have used drugs during sexual activity than the men at no risk. In each case, the differences in probability of drug use by risk category are highly significant. The proportionate increase in risk for dangerous sexual practices appears to be greater if the drug used during such activity is illegal (e.g., marijuana and "other drugs") rather than legal and easily available (e.g., alcohol and poppers). It is also important to point out that except in the case of "other drugs," the majority of men at high-risk combined drug use with sexual activity at least once during the previous month.

The relationship between the number of drugs used while having sex during the previous month and the risk score for AIDS is presented in Table 3. Those who used more than one drug during sex could either have been using drugs concurrently, or used one drug during a particular sexual encounter and another drug on a second occasion. The men who had sex without drugs were 4 times more likely to be at no risk than those who used three or more drugs. Conversely, the men who used three or more drugs during sex were 4.3 times more likely to be at high risk than those who had sex without drugs. Over half of the men who did not combine drugs with sex in the previous month had no risk. On the other hand, over half of the men who used three or more drugs while engaged in sexual activity during the same time period were at high risk. This relationship is highly significant.

We also examined the relationship between high-risk behavior and overall frequency of drug use with sex. These comparisons, not shown here, indicate a highly significant 
Table 2. Use of Drugs During Sexual Activity by AIDS Risk Scores, in Percentages

Drug Used During Sex Last Month:

\begin{tabular}{lcccc}
\cline { 3 - 4 } & $\begin{array}{c}\text { Alcohol } \\
(n=298)\end{array}$ & $\begin{array}{c}\text { Poppers } \\
(n=202)\end{array}$ & $\begin{array}{c}\text { Marijuana } \\
(n=218)\end{array}$ & $\begin{array}{c}\text { "Other Drugs" } \\
(n=101)\end{array}$ \\
\hline Risk Score for AIDS & & & & \\
No Risk & 38.5 & 25.6 & 25.6 & 10.3 \\
Medium Risk & 66.7 & 39.2 & 42.4 & 15.8 \\
High Risk & 79.4 & 66.0 & 65.7 & 35.4 \\
Chi square $=$ & 52.0 & 35.8 & 47.5 & 31.8 \\
$p=$ & .0000 & .0000 & .0000 & .0000 \\
\hline
\end{tabular}

Table 3. Number of Different Drugs Used during Sex by Risk Score for AIDS, In Percentages

\begin{tabular}{|c|c|c|c|c|}
\hline & \multirow[b]{2}{*}{$\begin{array}{c}\text { None } \\
(n=111)\end{array}$} & Number of Drugs & \multirow[b]{2}{*}{$\begin{array}{c}\text { Two } \\
(n=95)\end{array}$} & \multirow[b]{2}{*}{$\begin{array}{l}\text { Three or More } \\
\quad(n=155)\end{array}$} \\
\hline & & $\begin{array}{c}\text { One } \\
(n=100)\end{array}$ & & \\
\hline \multicolumn{5}{|c|}{ Risk Score for AIDS } \\
\hline $\begin{array}{l}\text { No Risk } \\
\text { Medium Risk } \\
\text { High Risk }\end{array}$ & $\begin{array}{l}56.8 \\
29.7 \\
13.5\end{array}$ & $\begin{array}{l}22.0 \\
51.0 \\
27.0\end{array}$ & $\begin{array}{l}10.5 \\
45.3 \\
44.2\end{array}$ & $\begin{array}{l}14.2 \\
27.1 \\
58.7\end{array}$ \\
\hline Total & $100 \%$ & $100 \%$ & $100 \%$ & $100 \%$ \\
\hline \multicolumn{5}{|c|}{ Chi square $=111.4, p=.0000$} \\
\hline
\end{tabular}

correlation between how often drugs are used with sex and sexual practices likely to transmit the AIDS virus.

Table 4 summarizes the relationship between drug use during sexual activity and change in risk scores from May, 1984 to May, 1985. Table 4 compares men who were at no risk as of May, 1984 to men at high risk at the same time $(n=311)$. By May of 1985, some of the men who were originally at no risk increased their risk scores for AIDS, similarly, some men who originally had high-risk scores for AIDS decreased risky sexual behavior. Each of these four distinct groups is compared according to current drug use behavior during sexual activity. These comparisons allow a measure of whether men who currently use drugs or alcohol during sexual activity have different histories of having adopted safe sex guidelines over the previous year.

In Table 4, the proportion of men who used drugs at least once during sexual activity during the month previous to May, 1985 is compared according to change in risk scores during the previous year. The men who were originally at no risk but who increased risk upon follow-up (row 2) were at least 2 times more likely to use alcohol, poppers, or marijuana during sexual activity than the men who remained at no risk (row 1). Furthermore, men who increased risk were nearly 7 times more likely to use "other drugs" than the men who remained at no risk. Similar findings emerge in a comparison of the men originally at high risk but who decreased risky sexual behavior (row 3) and those who remained at high risk (row 4). The men who re- 
Table 4. Prevalence of Use of Specific Drugs During Sex in May of 1985 by Change in Risk Scores from May of 1984-May of 1985

Used During Sex Last Month

\begin{tabular}{lcccc} 
& Alcohol & Poppers & Marijuana & "Other Drugs" \\
\hline $\begin{array}{l}\text { No Risk-May, 1984 } \\
\text { T1 No Risk }\end{array}$ & 32.6 & 17.4 & 20.0 & 4.3 \\
$\begin{array}{l}\text { T2 No Risk } \\
(n=46)\end{array}$ & & & & \\
$\begin{array}{l}\text { T1 No Risk } \\
\text { T2 More Risk } \\
(n=54)\end{array}$ & 77.8 & 37.0 & 46.3 & 29.6 \\
$\begin{array}{l}\text { High Risk-May, 1984 } \\
\text { T1 High Risk }\end{array}$ & & & & \\
$\begin{array}{l}\text { T2 Less Risk } \\
(n=95)\end{array}$ & 55.8 & 49.0 & 40.6 & 15.6 \\
$\begin{array}{l}\text { T1 High Risk } \\
\text { T2 High Risk } \\
(n=116)\end{array}$ & 75.9 & 64.7 & 67.2 & 36.2 \\
\hline
\end{tabular}

mained at high risk were approximately 1.5 times more likely to use alsohol, poppers, or marijuana during sexual activity than were the men who decreased risk.

Also of interest in Table 4 is a comparison of the men who remained at no risk with the men who remained at high risk. The men who remained at high risk were 2.3 times more likely to use alcohol during sexual activity. 3.7 times more likely to use poppers, 3.4 times more likely to smoke marijuana, and 8.4 times more likely to use "other drugs."

It is important to point out that a consistent pattern emerges from these data: men originally at no risk, but who increased risk over the previous year, are more likely to use drugs and alcohol during sexual activity than were the men who remained at no risk for AIDS. Men originally at high risk for AIDS, yet who managed to decrease risk over the past year, are less likely to use drugs and alcohol during sexual activity than the men who remained at high risk.

Change or stability in high-risk sexual practices over the past year is associated in a similar way with the number of different drugs used during sex and with the proportion of sexual encounters involving drugs. In both cases, low drug use is related to the maintenance of low-risk sex practices and to switching from high to low-risk behaviors.

\section{DISCUSSION}

Before the findings are summarized, it is important to keep in mind certain limitations of this data set. Although every attempt was made to secure a sample which reflected the broad diversity of the gay male community of San Francisco, it appears that the cohort initially contained an oversampling of gay men at high risk for AIDS. However, attrition from the sample as it was followed over time seems to have moderated this over-representation. Furthermore, it should also be kept in mind that these 
findings are drawn from a cohort based in the San Francisco Bay area. Generalizing these results to other communities of gay men in the United States may not be appropriate. Nonetheless, these data are probably a good measure of the direction and magnitude of the relationship between alcohol and drug use during sexual activity and the adoption of safe sex techniques among self-identified gay men who reside in urban settings.

The cross-sectional analysis revealed that use of particular drugs during sex, the number of drugs used during such activity, and the frequency of combining drugs and sex are all positively associated with risky sexual activity for AIDS. The retrospective data showed that men who currently abstain from drug use during sexual activity are most likely to have remained at no risk for the sexual transmission of HTLV-III virus over two measurement points during the previous year. The men who currently combined drug use with sex were most likely to have a history of high-risk sexual activity over the previous year. Larger proportions of men who increased risk used each of the four drugs than those men who had no risk at both measurement points. Smaller proportions of men who decreased risk from the previous years' measurement were currently less likely to combine any of the four drugs with sexual activity than those who stayed at high risk. These findings show a strong relationship between drug and alcohol use during sex and non-compliance with safe sex techniques to prevent the spread of AIDS.

About $90 \%$ of the men in the cohort correctly identified safe sex techniques as of May, 1984. Between May of 1984 and May of 1985, numerous health education programs were conducted by a variety of community- and government-based organizations. Saturation coverage of the epidemic by the local straight and gay media made it difficult for a member of the gay community to miss the messages of the health education campaigns. Nonetheless, a significant proportion of gay men in our cohort did not adopt safe sex practices as of May, $1985 .^{12}$ Our findings suggest that an important correlate of failure to practice safe sex is use of recreational drugs and alcohol during sexual encounters. To the extent that high-risk sexual behavior and drug and alcohol use are related, attempts to promote safe sex may need to include educational messages about drinking and drug use during sexual activity as well.

The cross-sectional and retrospective character of the data we have presented does not permit distinguishing between correlation and causation in the association between drug use and risky sex. There is nothing in these findings which proves conclusively that alcohol or drug use causes high-risk sexual behavior, or that attempts to control the use of alcohol and drugs in the gay community will reduce the incidence of HTLV-III infection. At present, the extent to which risky sex drives drug use, drug use drives risky sex, or some combination of both pathways exists, is not known.

Several competing hypotheses are available to explain the associations between alcohol and drug use during sexual activity and high risk sexual activity. These competing hypotheses should be carefully considered both for research efforts which further examine this association and for the design of health education and intervention programs undertaken to increase compliance with safe sex guidelines. As Ostrow ${ }^{15}$ has pointed out, these hypotheses might be labeled the "disinhibition," "aphrodisiac," "personality," "social context," "multifactorial," and "null" hypotheses. In Table 5, data sets needed to test these hypotheses and the health education and intervention implications of each are outlined. 


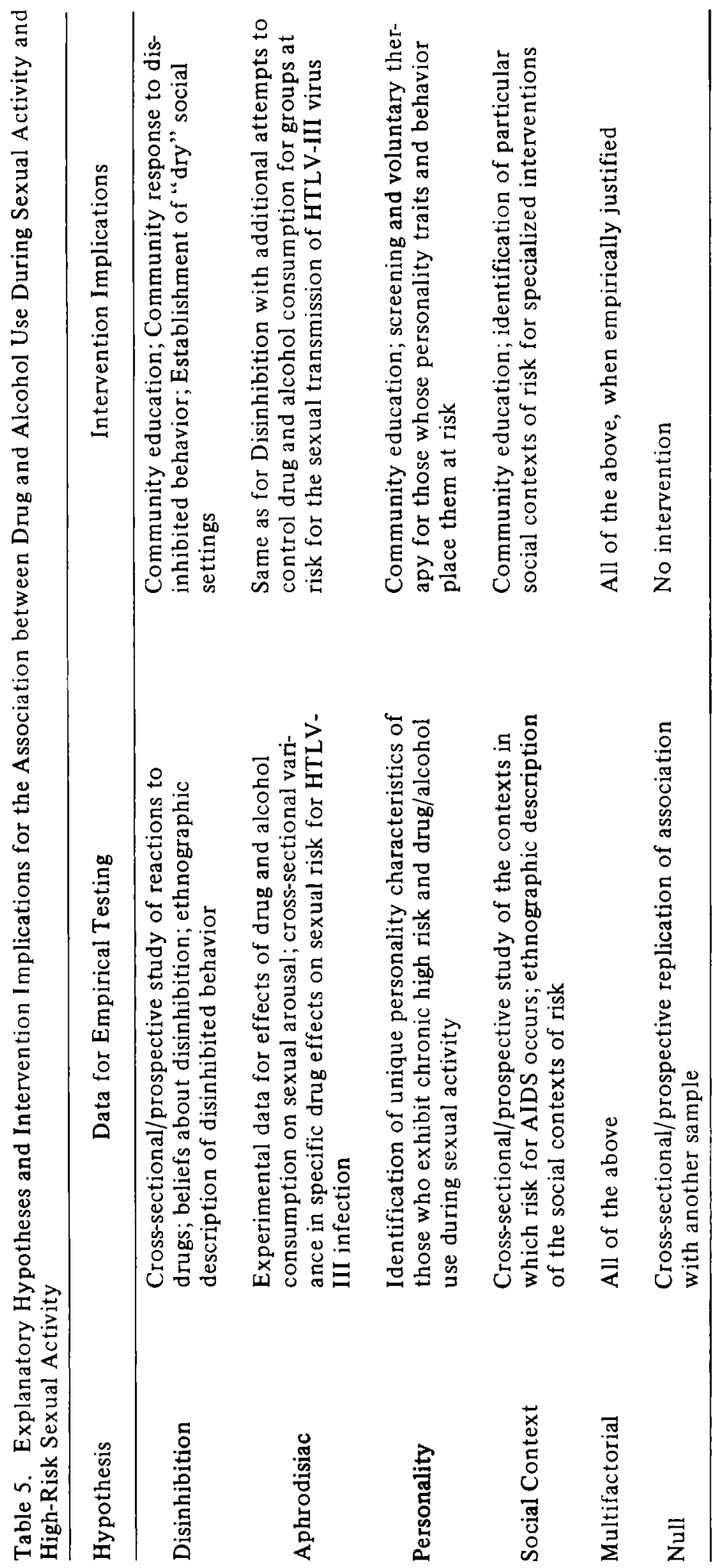


The "disinhibition hypothesis" asserts that culturally learned responses to substance use are causal of the content of inebriated behavior. As Room ${ }^{16}$ has described this idea:

"In recent years, evidence has been building up from a number of disciplinary areas to suggest that the link between alcohol and disinhibition is a matter of cultural belief rather than of pharamacological action. Alcohol is certainly a psychoactive drug: we feel different when drunk than when sober. But how we interpret those feelings, and in particular how we act on them, is largely determined by culture and circumstance: thus what is pharmacologically the same drug can make us aggressive or passive, ebullient or morose, frenetic or immobile. In this view, psychoactivity does not determine whether behavior is disinhibited or controlled: it simply provides an empty vessel of altered consciousness for culture, circumstance and personality to load with meanings and explanations."

A disinhibition hypothesis for the associations between substance use during sexual activity and high-risk sexual activity among gay men, then, would assert that within gay male culture the physiological effects of substance use are often interpreted in a way that encourages participation in high-risk sexual activity. Cross-sectional data to test this hypothesis would include the study of self-reported reactions of gay men to alcohol and drug use, particularly in "natural" (e.g., gay bar or social) settings and beliefs held by gay males about appropriate behavior when taking drugs or alcohol. In particular, the study of beliefs held by gay men about appropriate disinhibited behavior should focus on the attribution of blame for such comportment, that is, whether being drunk serves as an acceptable excuse for risky sexual behavior. Participant-observation ethnographic data on public drinking and drug use practices would also be a useful contribution to the interpretation of these survey data. Prospective analyses would treat each of the survey measures listed earlier as independent variables; that is, as reactions to intoxicants and/or beliefs about the proper use of substances change over time, the hypothesized consequent changes in high-risk sexual behavior could be measured. Should the disinhibition hypothesis be supported, several health education and intervention actions would appear to be warranted. The first of these would simply be widespread communication within the gay male community that combining sexual activity and substance use is associated with high risk sexual behavior. These communications should be made in such a way that disinhibition is discouraged as an acceptable excuse for risky sexual behavior. Indigenous response to the problem of disinhibited high-risk sexual behavior should be encouraged within the gay male community. In particular, community groups may wish to devise strategies which minimize the combination of courtship behavior and drug and/or alcohol use, perhaps by the establishment of safe places for gay men to meet socially which do not serve alcohol or allow drug use.

Another hypothesis which might explain the association between drug and alcohol use during sexual contact and high-risk sexual behavior would be that certain drugs and alcohol have an aphrodisiacal effect, e.g., that drug and alcohol use cause such intensity of libido through a physiological mechanism that control of sexual behavior is lost. This hypothesis can be challenged with data from this study by pointing out that the associations between alcohol, poppers, marijuana, and the "other drugs" were each significantly associated with increased sexual risk. That is it is unlikely that such disparate drugs as alcohol, marijuana, and poppers each have such a similar, and pro- 
found, effect on libido. Nonetheless, this relationship might be tested by utilizing balanced placebo experimental models already developed for alcohol use ${ }^{11}$ so that controls can be made for beliefs about alcohol or drugs in measuring their effects on sexual arousal. If the aphrodisiac hypothesis is supported, the implications for intervention are the same as for the disinhibition hypothesis, with the addition of further attempts to control drug and alcohol consumption among groups at risk for the sexual transmission of AIDS.

The personality hypothesis posits that the association between risky sexual behavior and drug and alcohol use during sexual behavior are artifacts deriving from underlying personality needs which demand the concurrent use of intoxicating substances and participation in sexual behavior likely to transmit the HTLV-III virus. Since these personality dynamics have yet to be identified, an indirect test of this hypothesis could be attempted by using prospective designs which examine the relationship between change in drug and alcohol use during sexual activity and change in participation in risky sexual behavior. Under the assumption that personality traits are strongly fixed by adulthood, men who exhibit concurrent change in recreational substance use during sexual activity and participation in high-risk sexual activity should be assumed not to have the personality risk factors which may underlie risk for exposure to HTVL-III virus. Even should a strong prospective relationship between change in sub. stance use during sexual activity and sexual risk for AIDS be established, however, it is to be expected that some individuals would exhibit chronicity for concurrent drug and alcohol use and high-risk sexual activity. If this is the case, then research efforts should be initiated to identify whether this chronic group can be characterized by a set of underlying personality characteristics likely to predispose towards risk taking, most effectively by the administration of a personality inventory instrument. If the chronic group is proportionately different from the rest of the men in the prospective cohort, then specific personality iraits can be hypothesized to be risk factors for exposure to HTVL-III virus. This hypothesis can be further tested (once such specific personality traits have been identified) through cross-sectional tests of association with risky behavior and continuing prospective tests. Should the personality hypothesis hold for certain types of gay men, intervention would include health education efforts to explain the implications of this relationship within communities at risk for sexual exposure to AIDS, along with community screening and voluntary therapy for those individuals who have the personality characteristics which place them at risk for exposure to AIDS.

The social context hypothesis posits that the combination of substance use with high-risk sexual activity for AIDS is a "... learned social ritual, embedded in a com. plex social milieu and strongly interrelated through a system of shared cues, exchanges, and interdependencies." 15 In short, this hypothesis suggests that certain subgroups of the gay male community have learned to combine high-risk sex with drug and/or alcohol use and have found this combination of behavior difficult to change, so that sexual expression is routinely high risk and routinely involves the use of drugs and/or alcohol. Cross-sectional study of self-reported contexts of high-risk sexual behavior could be used to test this hypothesis. Prospective study of the contexts of risk can be used to determine whether continuance or discontinuance in participation in such social contexts predicts change in risk status over time. Should the cross-sectional survey data support that this hypothesis holds for at least some groups of gay men, ethnographic data which describe the social contexts and rituals in which high-risk sexual activity 
are embedded would be most useful in designing insightful prevention strategies. Intervention campaigns to lower sexual risk within such groups are hampered by this purposeful combination of two types of presumably intractable behavior: sexual technique and recreational substance use. However, an attempt to prevent initiation into risky social contexts through health education efforts should probably be attempted. Further, in designing prevention strategies within existing "risky" social groups, it is probably advisable to emphasize the adoption of safe sex techniques rather than what may well otherwise be unproblematic drug or alcohol consumption. Safe sex techniques should probably be presented to such groups as additions to sexual repertoires, rather than as an attempt to curtail esteemed sexual practices.

The multifactorial hypothesis posits that the relationship between drug and alcohol use during sexual activity and high-risk sex is the result of a complex web of determinants, including those already listed and possibly others not yet identified. The necessity for adopting this hypothesis would be established by showing that any two of the hypotheses listed above are empirically supported. Given the complexity of drug and alcohol use behavior and sexual expression, it is likely that multifactorial explanations for this association will prove to be empirically supported. Research initiatives designed to test the hypotheses enumerated here, then, would be most productive if attempts are made to control for, and measure, interactive effects between factors governing risk. Interventions to manage the risk associated with the combination of this behavior should be comprehensive in nature, and should incorporate the appropriate intervention implications for each of the empirically-supported hypotheses.

The null hypothesis states that there is no relationship between recreational substance use during sexual activity and high-risk sexual activity. This hypothesis can be rejected by replication of the associations described in this paper with another cross-sectional sample of gay men, or, even more conclusively, by the replication of prospective associations between these two types of behavior. If the null hypothesis is supported, no health education or intervention efforts are justified.

Two recommendations seem warranted on the basis of the data presented in this paper. The first of these is that research should continue which attempts to establish which of the hypotheses already described can be empirically supported. As hypotheses are supported or rejected, results should be made immediately available to health educators so that effective strategies can be devised to help prevent the further spread of this virus. Secondly, although correlation is certainly not causation, the strength of the association between drug and alcohol use during sexual activity and high-risk activity is so strong (and the consequences of HTLV-III infection so profound) that health education efforts to communicate the fact of this association seem justified. In part, these findings are presented here so that interest will be generated among health educators and prevention workers in developing effective strategies which take into account recreational substance use during sexual activity as a condition under which compliance with AIDS health education guidelines is suspended. Experience gained in developing prevention and education strategies developed to meet this goal within the gay male community may well be of use in other communities, particularly if the incidence of veneral AIDS increases among heterosexuals. At present, educational campaigns should emphasize that the causal pathway behind this association is not yet understood, but that combining sexual activity and drug and alcohol use is associated with higher risk for HTLV-III infection. Further, these campaigns should be conducted to undercut the notion that inebriation excuses behavior-whether participants com- 
bine recreational substance use with sexual activity or not, high-risk sexual activity can have tragic results.

Until such time that a vaccine or cure for AIDS is developed, the primary means of managing this epidemic is through health education. The fact that the principal risk group for AIDS is a socially stigmatized population which has responded to the traditional discrimination of mainstream society by constructing an "underground" social world, and the fact that most risk behavior for AIDS occurs in settings beyond reach of regulatory agencies, have placed additional importance on health education efforts. Health educators have learned to communicate the safe sex guidelines in terms which are culturally meaningful, and it is clear that the vast majority of gay men in San Francisco (where resources have been devoted to innovative health education efforts) understand the purpose and specifics of these guidelines. However, increasing attention should be devoted to identifying the circumstances under which compliance with safe sex guidelines is suspended. The data in this article suggest that the use of drugs and alcohol during sexual activity may be one such circumstance.

This article could not have been written without the participation of the respondents for "The AIDS Behavioral Research Project." The men who participated in this study patiently filled out questionnaires concerning sensitive lifestyle issues over a three-year period in the hope that their responses might help control the AIDS epidemic. Hank Wilson assisted in the design of the alcohol and drug measure. The authors would like to thank Diane Davis of the Medical Research Institute of San Francisco, Alcohol Research Group and Glenn Saika, Division of General, Internal Medicine, University of California, San Francisco for their programming assistance. Drs. Robin Room and Kaye Fillmore of the Alcohol Research Group provided valuable critiques of an earlier draft of this paper, as did two anonymous reviewers. This article was supported in part by a grant from NIMH (MH39553) and a contract with the National Institute on Alcoholism and Alcohol Abuse (AA05595).

\section{References}

1. McKusick L, Horstman W, Coates TJ: AIDS and sexual behavior reported by gay men in San Francisco. Am J Pub HL 75(5): 493-496, 1985.

2. McKusick L, Wiley JA, Coates TJ, Stall R, Saika G, Morin S, Charles K, Horstman W, Conant MA: Reported changes in the sexual behavior of men at risk for AIDS, San Francisco, 198284 - The AIDS behavioral research project. Pub HL Rep 100: 622-625, 1985.

3. Fifield LH, Latham JD, Phillips C: Alcoholism in the Gay Community: The Price of Alienation, Isolation and Oppression. A Project of the Gay Community Services Center. Los Angeles, CA 1977.

4. Lohrenz LJ, Connelly LC, Coyne L, Spare KE: Alcohol problems in several midwestern homosexual communities. J Stud Alcohol 39: 1959-1963, 1978.

5. Morales ES, Graves, MA: Substance Abuse: Patterns and Barriers to Treatment for Gay men and Lesbians in San Francisco. San Francisco Community Substance Abuse Services. San Francisco: Prevention Resource Center, 1983.

6. Brown S, Goldman M, Inn A, Anderson L: Expectations of reinforcement from alcohol: their domain and relation to drinking patterns. J Consul Clin Psychol 48: 419-426, 1980.

7. Briddell DW, Wilson GT: The effects of alcohol and expectancy set on male sexual arousal. J Abnorm Psychol 85: 225-234, 1976.

8. Farkas G, Rosen, RC: The effects of ethanol on male sexual arousal. $J$ Stud Alcohol 37 : 265-272, 1976.

9. Malatesta VJ, Pollack RH, Wilbanks WA, Adams HE: Alcohol effect on the orgasmic-ejaculatory response in human males. J Sex Research 15: 101-107, 1979.

10. Briddell DW, Rimm DC, Caddy GR, Krawitz G, Shous D, Wunderlin RJ: Effects of alcohol and cognitive set on sexual arousal to deviant stimuli. $J$ Abnorm Psychol 87: 418-430, 1978. 
11. Wilson GT, Lawson DM: Expectancies, alcohol and sexual arousal in male social drinkers. $J$ Abnorm Psychol 85: 587-594, 1976.

12. Research and Decisions Corporation: Designing an effective AIDS prevention campaign strategy for San Francisco: Results from the first probability sample of an urban gay male community. Report prepared for the San Francisco AIDS Foundation. San Francisco, 1984.

13. Anderson RE, Levy J: Prevalence of antibodies to AIDS-associated retrovirus in single men in San Francisco. Lancet 8422:217, 1985.

14. McKusick L, Coates TJ: Behaviors associated with receiving and transmitting the HTLV-III virus among gay men in San Francisco: the AIDS behavioral research project. Paper presented at the American Psychological Association Annual Meetings. August, 1985.

15. Ostrow, D: Barriers to the acknowledgementof the role of drugs and alcohol in AIDS and the treatment of drug and alcohol problems in persons with AIDS and HIV infection. Paper presented at the Joint Meeting of the American Medical Society on Alcoholism and Other Drug Dependencies and the Research Society on Alcoholism. April, 1986.

16. Room, R: Introduction p. v: Alcohol and Disinhibition: Nature and Meaning of the Link. R. Room and G. Collins, editors. Proceedings of a Conference Feb. 11-13, 1981. NIAAA Research Monograph No. 12, 1983. 